\title{
Vascular malformations: an update on imaging and management
}

\author{
Sergio Sierre, M.D. ${ }^{a}$, DaríoTeplisky, M.D. ${ }^{a}$ and José Lipsich, M.D. ${ }^{a}$
}

\begin{abstract}
Vascular malformations comprise a broad and heterogeneous range of lesions that often represent a diagnostic and therapeutic challenge for the pediatrician. For a long time, the use of an inaccurate nomenclature has led to confusion. Since management depends on the specific vascular malformation, a proper classification and identification is critical.

The objective of this article is to provide the necessary information about the current classification and terminology of vascular anomalies, including basic concepts about available imaging diagnostic and therapeutic tools for the management of such complex condition.

Key words: vascular malformations, diagnosis, lymphaticabnormalities, arteriovenousmalformation, sclerotherapy, therapeutic embolization.
\end{abstract}

http:/ /dx.doi.org/10.5546/aap.2016.eng.167

\section{INTRODUCTION}

Vascular anomalies include vascular tumors and malformations. Frequently, they are incorrectly called "angiomas" or "hemangiomas", and comprise a broad range of lesions, from a simple "birthmark" to lifethreatening conditions. In general, they affect newborn, infants, young children and young adults. Vascular anomalies are diffuse or localized defects occurring during the embryonic phase and may involve

a. Department of Interventional Radiology. Hospital de Pediatría "Dr. Prof. Juan P. Garrahan". Buenos Aires, Argentina.

E-mail address: Sergio Sierre, M.D.: sergio.sierre@usa.net

Funding:

None.

Conflict of interest: None.

Received: 07-13-2015 Accepted: 08-06-2015 any part of the body. They usually develop between the fourth and tenth week of gestation. These anomalies are present at birth, but may not be evident until weeks, months or years later. $^{1-4}$

\section{CLASSIFICATION}

In spite of multiple efforts for the diffusion and usage of a standard classification, nomenclature and terminology are still confusing for general pediatricians..$^{3-7}$

The terms "hemangioma" or "angioma" are frequently used in everyday clinical practice to refer to either vascular malformations or vascular tumors, although their etiology, natural course and treatment are different.

In 1982, Mulliken and Glowacki described the most widely accepted classification, which became the basis for naming and studying these lesions. It is a biological classification based on the presence of cell proliferation, histological features, and clinical findings. ${ }^{8}$

This classification divides these anomalies into vascular tumors and malformations.

- Vascular tumors: hemangiomas are the most common type. They are benign tumors that may not be apparent at birth; they present increased cell proliferation and hyperplasia, and are characterized by an early proliferation phase and a subsequent involution stage. . $^{3,6-8}$

- Vascular malformations: they are present at birth. They are characterized by dysplastic vascular channels with normal cell proliferation. They grow at the same rate as the child and present no spontaneous involution. Vascular malformations are further classified based on the vascular channel components involved: arterial, venous, lymphatic and capillary, or a combination of these. Their clinical features define their onset, follow-up and specific management. . $^{2-4,8}$

In 1993, Jackson et al. proposed a radiological classification based on flow and hemodynamics. They classified vascular malformations as high-flow (arteriovenous fistula, arteriovenous malformation) or low-flow (capillary, venous, lymphatic malformations) ${ }^{9}$ Any vascular malformation involving an arterial channel 
will be considered "high-flow", while any malformation without an arterial channel will be deemed "low-flow". 2,7-10

In 1996, the International Society for the Study of Vascular Anomalies (ISSVA) embraced and extended this classification, which has now become the most widely used system.

Conceptually, the ISSVA defined two types of anomalies, vascular malformations (which will be analyzed in this article) and vascular tumors (being hemangioma the most common type) (Table 1).

In 2014, the ISSVA expanded this classification based on ongoing research and updates on this topic. ${ }^{11}$ Vascular malformations are divided into simple and combined, and a section on the association with other anomalies and syndromes has been introduced (Table 2), among others. This new classification is available at www.issva.org.

\section{CLINICAL EXAMINATION}

In clinical practice, there are vascular malformations with a typical clinical course, clear characteristics on physical exam that require no imaging studies for diagnosis, while others are atypical, deep lesions covered by normal skin or involving vital structures for which clinical diagnosis is unclear or insufficient. Imaging studies are essential for an accurate diagnosis, to make a specific management decision and to discuss the prognosis with parents.

Recent scientific and technological advances in the field of imaging studies, surgery and interventional radiology offer multiple resources to diagnose and treat these malformations.

In this article we will focus on currently available tools used to diagnose and manage this complex condition.

\section{VASCULAR MALFORMATIONS DURING CHILDHOOD}

These are localized vascular defects occurring during development, and they are basically classified by the type of channel components involved (arterial, capillary, venous or lymphatic) and the malformation hemodynamics (high-flow or low-flow). ${ }^{1-3,8,11}$

They may grow at the same rate as the child, never going away or involuting. Many of these malformations become apparent during puberty because they have hormone receptors that make them sensitive to estrogen and testosterone variations. ${ }^{11,12}$

\section{Low-flow malformations Venous malformations}

These are the most common type. They involve an abnormally developed vein wall that affects vein function. Involved channel

TABLE 1. Classification of vascular malformations/International Society for the Study of Vascular Anomalies, 1996

\begin{tabular}{l|cc}
\hline Vascular tumors & & Vascular malformations \\
\hline Hemangiomas and others & Simple & Combined \\
\hline & Capillary & Arteriovenous fistula \\
& Lymphatic & Arteriovenous malformation \\
& Venous & Capillary-venous, capillary-lymphatic-venous malformation \\
& Arterial & Lymphatic-venous, capillary-lymphatic-venous malformation \\
& & Capillary lymphatic arteriovenous malformation \\
\hline
\end{tabular}

TABLE 2. Vascular malformations associated with other anomalies

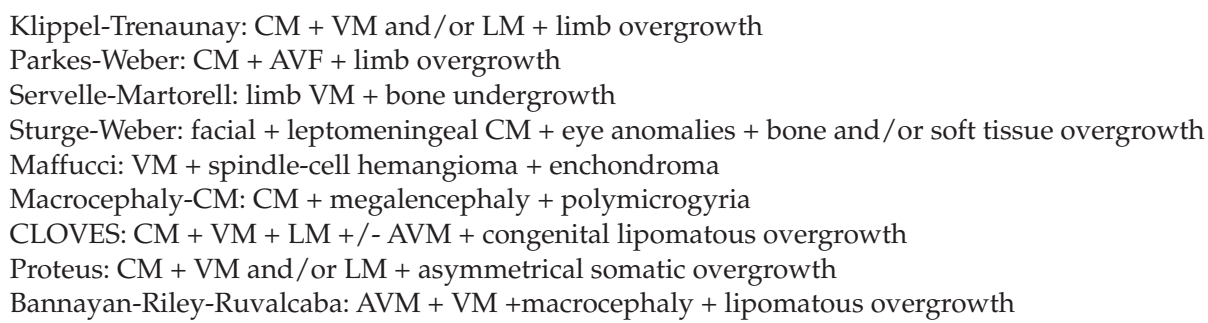

CM: capillary malformation; VM: venous malformation; LM: lymphatic malformation; AVF: arteriovenous fistula. AVM: arteriovenous malformation; CLOVES: congenital lipomatous overgrowth, vascular malformations, epidermal nevi, skeletal/scoliosis and spinal abnormalities. 
components are progressively dilated, which results in blood stagnation, leading to thrombosis, inflammation and pain. Venous malformations (VMs) frequently develop spontaneous localized thrombosis and thrombolysis. Persistent thrombi may calcify and phleboliths may be formed, typical of these lesions. $1,3,7,11,12$

In general, they are isolated and mostly develop in the cervicofacial region, limbs and trunk.

\section{Clinical presentation}

Clinical presentation is highly variable. Superficial lesions are soft, bluish, compressible and non-pulsatile; they may be flat, slightly elevated or markedly exophytic (Figure 1).

Lesions may be localized, well-defined or extensive, diffuse and infiltrating. On palpation, a compressible, sometimes tender mass is often found, especially when there are recently-formed clots or phleboliths. Many times, spontaneous pain occurs, especially in relation to the onset of intralesional clots. ${ }^{3,4,12,13}$

These malformations may grow in size depending on the patient's position (e.g., with the head down), with the Valsalva maneuver, during infant crying, or with compression. They may grow suddenly after an incomplete surgical resection, a biopsy, a trauma and/or infections. ${ }^{13,16}$ Sometimes, pain is attributed to joint, tendon or muscle involvement. These lesions may even get worse during menstrual periods or puberty ${ }^{13,15}$ (Figure 2).

Extensive VMs may present localized intravascular clotting due to the above mentioned intralesional thrombosis; they occur accompanied by hypofibrinogenemia and elevated D-dimer levels. It is important to know the patient's basic coagulation parameters before any intervention. $12,14,17$

VMs may be associated with other clinical conditions. Klippel-Trenaunay syndrome typically has capillary malformations, venous and lymphatic (macro- and/or microcystic) malformations, and soft tissue and bone hypertrophy. Generally, these lesions affect the lower limbs, and sometimes they may be bilateral or involve the upper limbs. ${ }^{10,12,18}$

\section{Imaging assessment}

For all vascular anomalies, Doppler ultrasound and magnetic resonance imaging (MRI) are generally the preferred methods for an adequate and complete assessment and follow-up of patients.
FIGURE 1. Venous malformation

A.

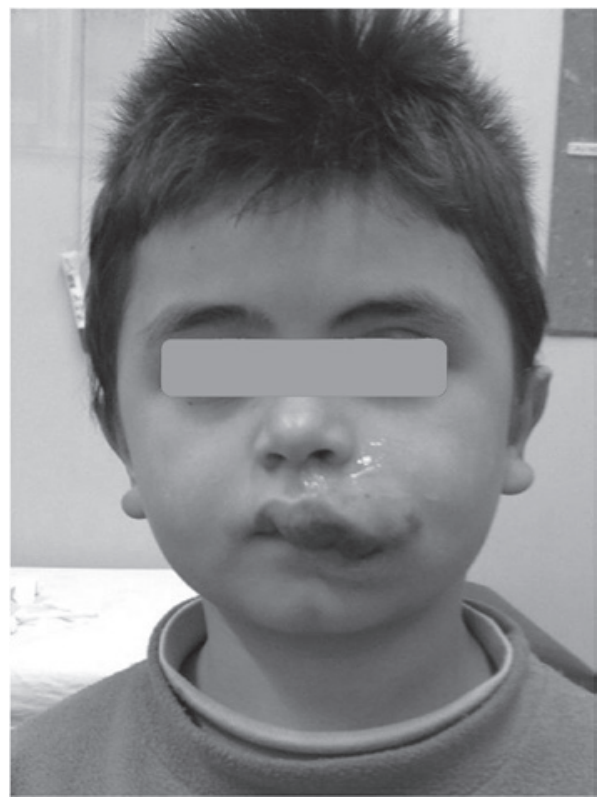

Six-year old male patient with a venous malformation of the upper lip.

B.

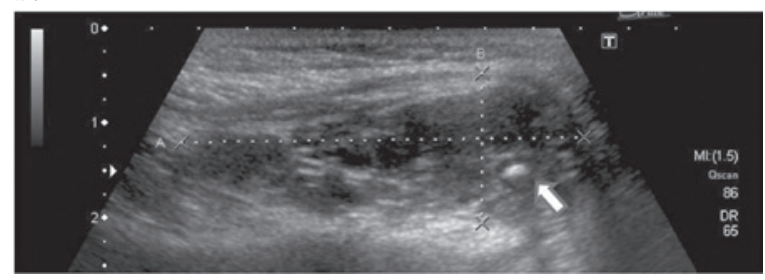

Ultrasound, longitudinal section, at the level of the lip. Heterogeneous pattern, with a phlebolith inside (arrow).

C.

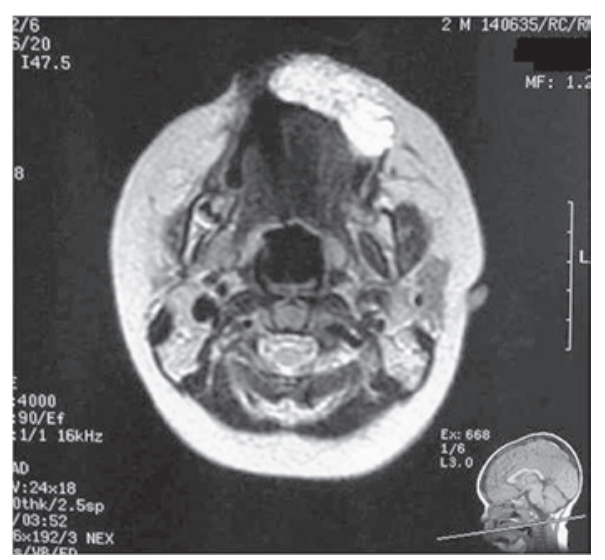

Magnetic resonance imaging of the same patient, axial T2 image showing hyperintense signal at the level of the lip, typical of venous malformations. 
In relation to VMs, the initial assessment is a clinical exam. Small, superficial lesions that are clearly diagnosed may not require imaging studies; however, most patients will require at least a Doppler ultrasound.

Ultrasound: Two patterns may be identified. A cavitary pattern (the most common type), which shows heterogeneous images with mixed echogenicity. Phleboliths may be present, and thus reinforce the diagnosis of a venous malformation. The other is a dysplastic pattern with multiple tortuous dilated veins infiltrating subcutaneous fat, muscles, tendons and adjacent tissues ${ }^{10,14,19}$ (Figure 1). These are low-flow lesions, so a Doppler ultrasound would be able to confirm a low-rate monophasic venous flow or the absence of flow within the malformation. Sometimes, flow may be observed when performing the Valsalva maneuver or compression (Figure 2).

Magnetic resonance imaging: An MRI allows to assess the extent of a lesion and its relationship to adjacent organs and structures. Recommended sequences are T1 (pre- and post-contrast) and T2 weighted images, with fat saturation. Typically, images may have an intermediate signal intensity in $\mathrm{T} 1$ and a hyperintense signal in T2 in relation to its content or the presence of hemorrhage or thrombosis. A gadolinium injection usually shows a diffuse enhancement of venous channels, unlike lymphatic malformations, which generally do not have this kind of enhancement. With T2 weighted images, phleboliths appear as focal areas of hypointense signal. $13,16,20$

Arteriogram: It is not indicated.

Percutaneous phlebography: If treatment is required for the lesion, this is the imaging method of choice to observe the hemodynamics and angioarchitecture of the malformation. This invasive procedure is reserved as an immediate step prior to percutaneous management.

\section{Treatment}

Small, asymptomatic lesions can be managed conservatively. Compression stockings may be used by patients with limb involvement. The main indication for treatment is pain. In other cases, in spite of the absence of pain, treatment is indicated if there is joint involvement, functional impairment or for aesthetic reasons. ${ }^{4,13,17,21}$

An effective medical treatment has not yet been discovered. In ongoing studies, it has been demonstrated that angiogenesis inhibitors have clinical effectiveness. ${ }^{22}$

In patients with extensive venous malformations, coagulation should be assessed in depth. Alterations in the muscle wall of the affected vein leads to blood stagnation and thrombi formation. In some cases, localized intravascular coagulation (LIC) may occur, which

\section{FIGURE 2. Venous malformation}

A.

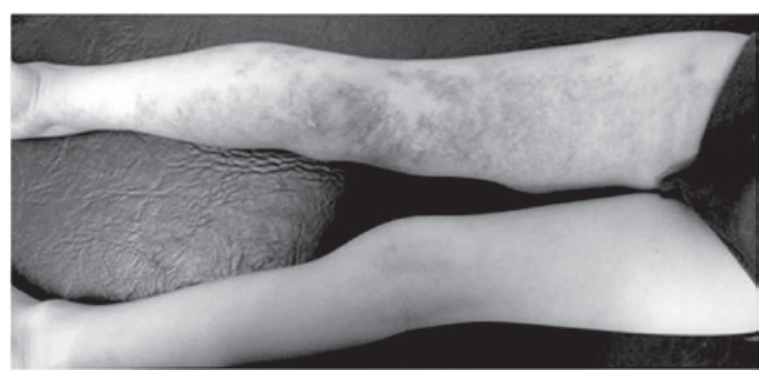

Thirteen-year-old female patient with an extensive venous malformation in the left lower limb (patient in ventral decubitus position).

B.

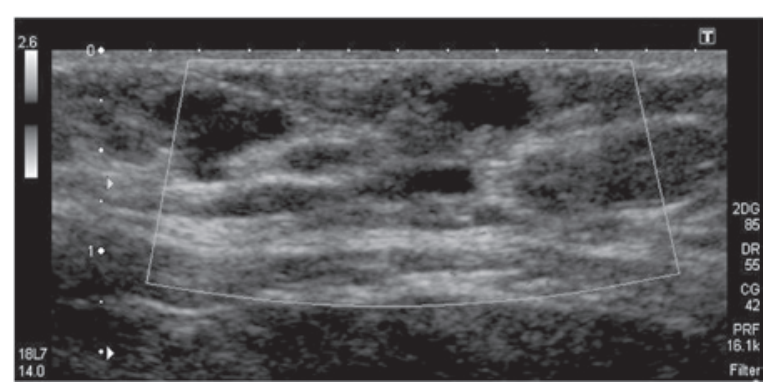

Doppler ultrasound of thigh region showing a cavitary pattern with no flow inside.

C.

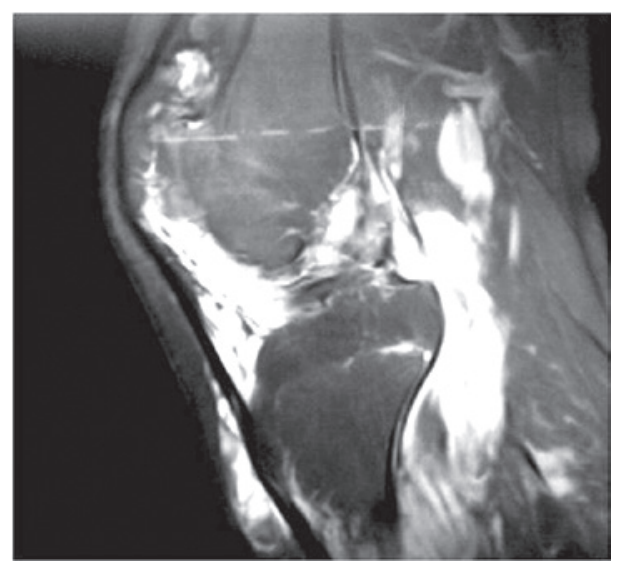

Sagittal T2 MRI of the left knee showing a hyperintense signal caused by a venous malformation that diffusely involves the intra-articular and peri-articular region. 
is translated into elevated D-dimer levels and reduced fibrinogen.

At referral centers, LIC is managed with low-molecular-weight heparin. Sclerotherapy is contraindicated until LIC is resolved due to the risk of generalized intravascular coagulation. ${ }^{12-14}$

Sclerotherapy is, at present, the treatment that has shown the best results. It is performed at the Interventional Radiology Unit, usually under general anesthesia and with strict monitoring of vital signs. Percutaneous treatment consists of injecting sclerosing agents (sodium tetradecyl sulfate, ethanol and bleomycin, among other common agents) that cause endothelial damage and therefore lead to inflammation and fibrosis. ${ }^{2,7,13,14,17}$ Many times, treatment is combined with additional surgical procedures.

In children with extensive head and neck malformations that involve the airways, it is necessary to consider the possibility of performing a tracheostomy prior to sclerotherapy or to have the child in mechanical ventilation for $48 / 72$ hours after the procedure because the inflammatory response may collapse the airways. For any of these cases, management is the result of a multidisciplinary approach. ${ }^{23-25}$

\section{Lymphatic malformations}

These are the second most common type of malformations. Lymphatic malformations are sequestrated and dilated lymphatic sacs that lack connection with the peripheral lymph system. The rate of these lesions is similar in both sexes. They may classified as macrocystic, microcystic, combined and mixed. $2,7,26$

Although there are multiple ambiguous definitions, a lesion is considered macrocystic if the lesion can be successfully aspirated (and subsequently slerosed), which reduces its size. This process is more complex in microcystic lesions. ${ }^{2,4,14}$

These malformations are more commonly located in the head and neck (70-80\%), usually in the posterior cervical triangle ${ }^{1,3,26}$ (Figure 3).

The remaining $20 \%$ may be located in the axillary, superior mediastinal, mesenteric, retroperitoneal areas, the pelvis and the lower limbs. They may be associated with Turner syndrome, Noonan syndrome, multiple pterygium, and certain trisomies. ${ }^{18}$

\section{Clinical presentation}

Most cases are detected around two years old, although some may be apparent at birth and even get diagnosed during an antenatal ultrasound. ${ }^{2,3,7}$
These malformations appear as palpable tumors with a soft consistency and a temperature similar to the rest of the skin. Their clinical appearance is varied and depends on their size and, especially, their location and depth. Lymphatic malformations grow naturally at the same rate as the patient. During adolescence, the size and symptoms of these lesions may increase, probably in relation to hormone receptors present in them. ${ }^{26}$

In general terms, changes in skin color and/or small blisters may occur, which are characteristic of these lesions, or they may even have healthy skin.

A sudden increase in volume may be the result of an infection or an intracystic hemorrhage. Superinfected lesions are tense, warm and erythematous masses; an empiric antibiotic therapy is indicated in these cases. ${ }^{27}$ Intracystic hemorrhage is less common, although it may be secondary to a trauma or occur spontaneously. The lesion's size increases suddenly, with a change in color and bruising, and less frequently, with an elevation in local temperature.

\section{Imaging assessment}

Ultrasound: Macrocystic lesions show multiple images of different size cysts, separated by echogenic septa. In case of bleeding, echogenic cysts or air-fluid levels are observed (Figure 3).

Microcystic lesions appear as echogenic images with ill-defined borders. Mixed lesions show cystic images inside an echogenic area. No flow is observed inside cysts, but vascularization may be evident in the septa separating them. Microcystic lesions lack blood flow. ${ }^{10,19,27}$

Magnetic resonance imaging: An MRI allows to assess the extent and depth of these lesions and their relationship to adjacent structures, as well as to observe their extension into the chest and airway involvement in the case of head and neck malformations. Macrocystic lesions show hyperintense signal in T2 images and low intensity signal in T1 images, with post-contrast enhancement of the septa. Microcystic lesions generally appear as T2 images with homogeneous hyperintense signal. ${ }^{4,10,16,20}$

\section{Treatment}

It has been demonstrated that medical treatment with antiangiogenic agents, such as rapamycin or sirolimus, is effective in very extensive, diffuse malformations, especially in newborn infants with extensive cervicofacial lesions. At present, there are different ongoing clinical studies with promising results for 
these specific indications. ${ }^{22}$ In many cases, this treatment is usually associated with percutaneous management of macrocystic lesions or even an additional surgical resection. ${ }^{22,28}$

Treatment with other drugs, such as sildenafil, has not proven to be safe and effective for this indication. ${ }^{29}$

Sclerotherapy is usually indicated as a first-line option for patients with macrocystic lesions. $4,7,14,17,30$ It is performed at the Interventional Radiology Unit, usually under general anesthesia. The same precautions considered for VMs should be taken into account in the case of malformations involving the airways.

The most commonly used sclerosing agents are tetradecyl sulfate, doxycycline and alcohol for macrocystic lesions. Acceptable results have been observed with bleomycin for microcystic malformations. ${ }^{2,4,7,14,17}$

In some cases, it is necessary to complete treatment with surgical resection of the remaining lesions. ${ }^{10,14,17,30}$
A.

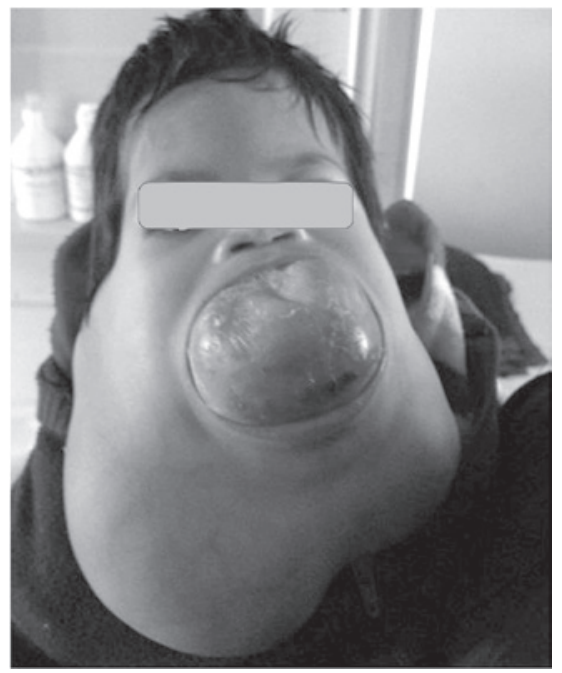

Eighteen-month-old male patient with an extensive mixedtype lymphatic malformation that involves the cervicofacial region, especially the tongue.

B.

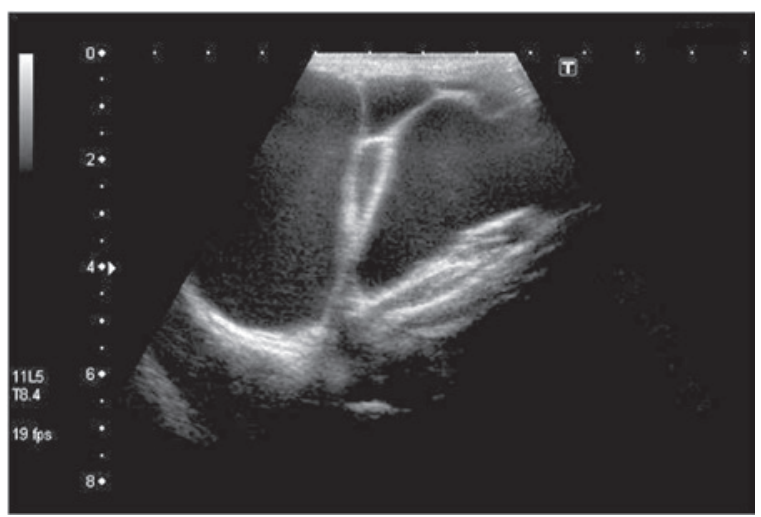

Sagittal ultrasound image of the neck showing the typical pattern of a macrocystic lymphatic malformation. Large cysts with fluid content (lymph) and thin walls identified.
C.

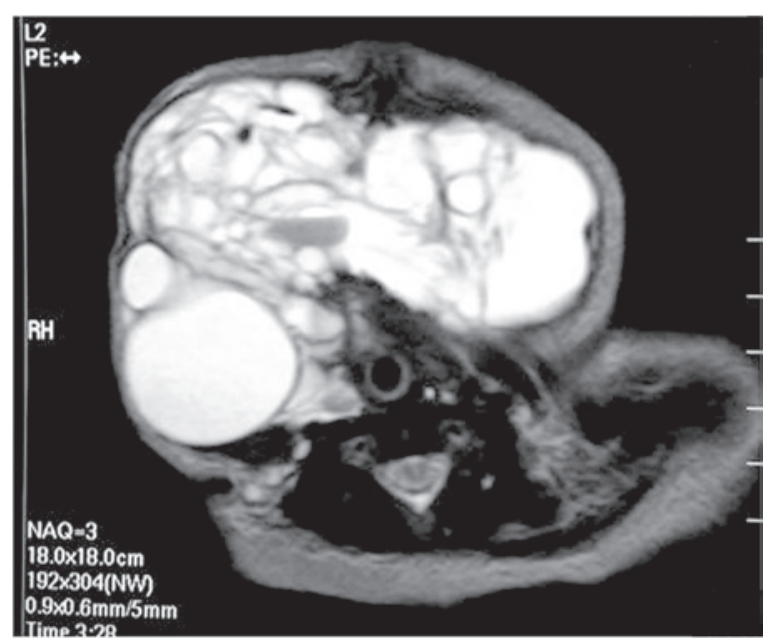

D.

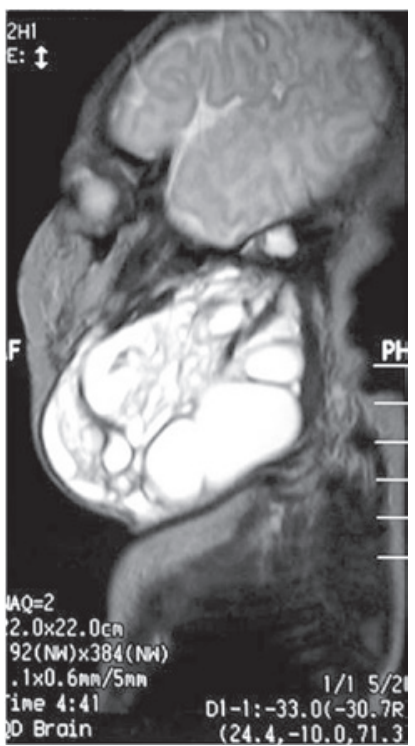

Magnetic resonance images. Axial (C) and sagital (D) T2 of the neck showing a hyperintense signal caused by the hight protein content and multiple septa. 


\section{Capillary malformations}

"Capillary malformation" (CM) is a generic term used to describe the presence of a "vascular stain" on the skin rather than to indicate a specific lesion. The most frequently recognized type of $\mathrm{CM}$ is a port-wine stain or nevus flammeus. This term also encompasses other types of vascular skin lesions, such as telangiectasias and hyperkeratosic macules. ${ }^{1,3,31}$

CMs affect the capillary network vessels of the skin and mucous membranes, which are made up of ectatic dermal capillaries, and increase with age. These lesions are frequently associated with focal hypertrophy of the bones and underlying soft tissues. ${ }^{31}$

CMs may be single and isolated, and may result in a severe aesthetic compromise. They may also be the expression of extracutaneous manifestation. CMs are present at birth, and no differences have been observed in their rate between both sexes. They are often located in the head and neck, and also in the trunk and limbs. $3,18,31,32$

CMs are frequently combined with other malformations (capillary-venous, capillaryarteriovenous, or capillary-lymphatic malformations) and they may even be a component of more complex anomalies, such as SturgeWeber syndrome, Parkes-Weber syndrome, the macrocephaly-CM complex, or hereditary hemorrhagic telangiectasia. ${ }^{11,18,32,33}$ In the SturgeWeber syndrome, CMs affect mainly, but not exclusively, the upper lids and the supraorbital region, and are frequently associated with seizures. ${ }^{33}$

Imaging studies for CMs are ultrasound and MRI, which only show a non-specific thickening of the dermis, occasionally extended to the subcutaneous cellular tissue. . $^{1,3,10}$ The study of choice in patients suspected to have SturgeWeber syndrome is an MRI. A brain MRI with intravenous contrast would be able to show soft tissue and adjacent bone hypertrophia, focal brain atrophy, leptomeningeal enhancement and thickening and the typical intracranial calcifications, among other findings. ${ }^{34,35}$

The only treatment option for CMs is laser, a technique most often effective. Among the different types of laser, pulse dye laser (PDL) is the standard treatment, especially for children. ${ }^{36,37}$

\section{High-flow malformations}

Arteriovenous malformations

This group mostly refers to two anomalies: arteriovenous malformations and fistulas. 3,7,11

Arteriovenous malformations (AVMs) are potentially more aggressive. They may be defined as lesions whose structure shows abnormal connections between arteries and veins, bypassing the capillary system. Such abnormal connections make up the malformation "nidus". These lesions are less common and their prognosis is less predictable given their usually difficult management. . $^{3,738}$

They are present at birth and may be asymptomatic for years. AVMs may become apparent as a result of trauma or during puberty due to hormonal changes.

\section{Clinical presentation}

Symptoms are related to hypervascularization, the presence of abnormal arteriovenous shunts, venous hypertension and tissue ischemia. Symptoms may include pulsatile masses, generally with increased skin temperature and palpable thrill. During auscultation, superficial lesions usually indicate a murmur. They may be accompanied by bone and soft tissue overgrowth and/or be associated with osteolytic lesions. ${ }^{1-4,7,14}$ In advanced stages, AVMs may have trophic changes due to venous hypertension, and distal ischemia due to vascular steal syndrome. Some cases are associated with congestive heart failure due to right ventricular overload generated by the high flow of these lesions ${ }^{12,39}$ (Figure 4).

\section{Imaging assessment}

Ultrasound: An ultrasound shows a heterogeneous, ill-defined mass with vascular dilations in and around the mass. Doppler ultrasound recognizes numerous vessels with a high arterial flow, a high diastolic peak, turbulent flow and pulsatile venous flow. 10,19,38

Magnetic resonance imaging: An MRI allows to assess vascular anatomy, the extent of the lesion and its relationship to adjacent structures. With T1 images, it is possible to recognize tubular structures with hypointense signal (flow void phenomenon), which usually represent afferent arteries and the malformation nidus, which reflects a high flow. Angio-MRI images are very useful, especially in children, because they show the lesion's afferent and efferent vessels and help to plan an eventual endovascular procedure. Additionally, an MRI is a very useful follow-up method after a procedure. ${ }^{3,4,10,16,20}$

Arteriogram: This invasive study shows the exact details of the AVM angioarchitecture and 
hemodynamics, afferent arterial pedicles, nidus and type of venous return, all critical to plan an endovascular management. Given that this is an invasive procedure, it is usually reserved as an immediate step prior to an endovascular procedure. ${ }^{3,7,14,30}$

\section{Treatment}

The treatment of choice of AVMs is endovascular embolization. This procedure is a challenge for the treating team and requires a deep understanding of available techniques and of the vascular anatomy of these lesions.
Transarterial occlusion of afferent vessels that supply the AVM should be particularly careful and occlusion of normal arterial vessels that feed other organs or tissues should be avoided. It should be an intra-nidus occlusion, without exception. ${ }^{38,41}$ If large-caliber draining veins are predominant in the AVM, transvenous endovascular embolization and subsequent occlusion of draining veins are an alternative approach because it has been shown that venous occlusion has a coadjuvant effect in nidus thrombosis. Another alternative would be to directly approach the malformation nidus by a direct percutaneous fine-needle puncture.
A.

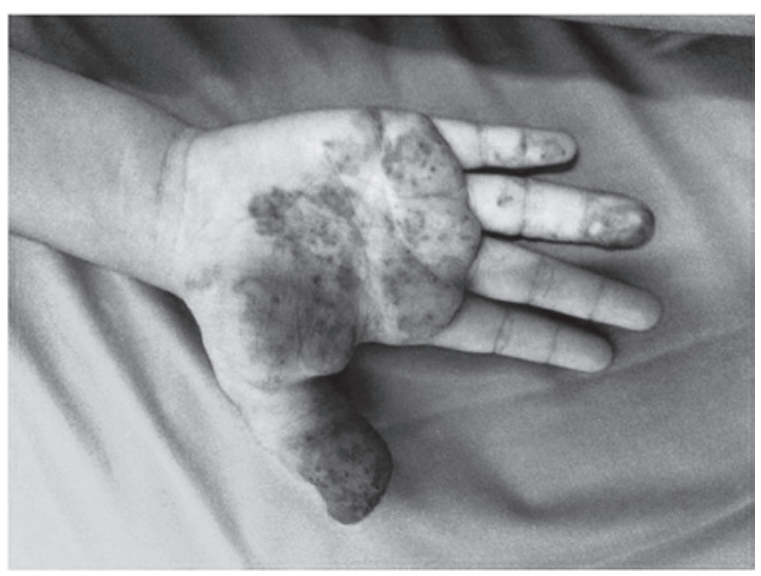

Six-year-old female patient with an arteriovenous malformation in her right hand, with involvement of the palm, thumb, ring finger and little finger.

B.

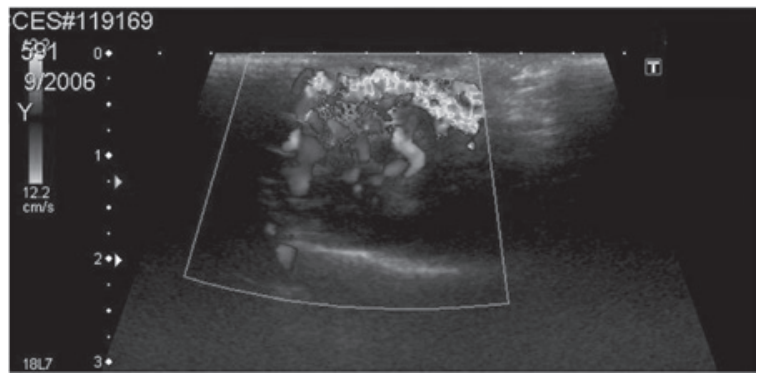

Color Doppler ultrasound showing increased vascularization and turbulent flow in the palm of the hand.
C.

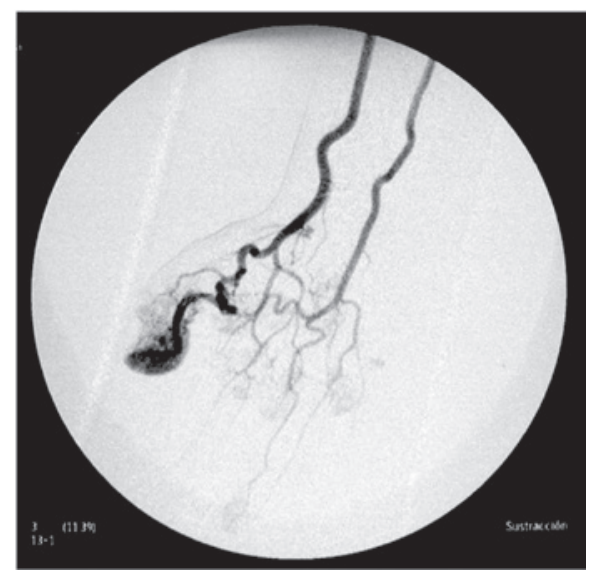

Arteriogram of the right hand.Early arterial phase showing the afferent vessels of the arteriovenous malformation and rapid hypervascularization of the thumb.

D.

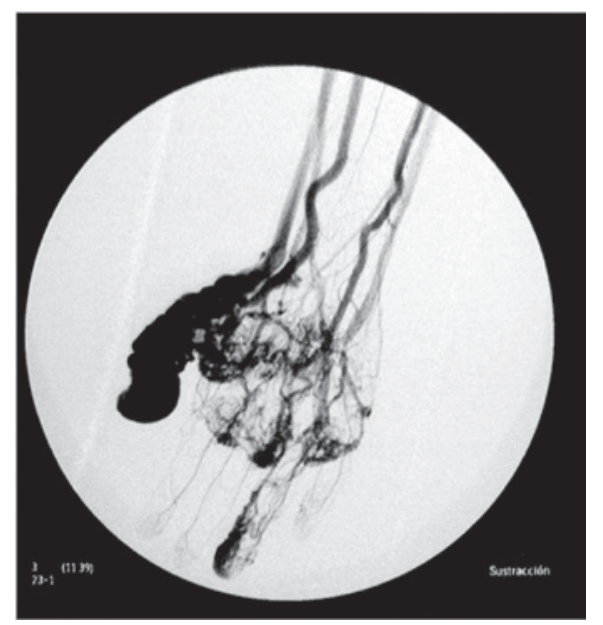

The typical nidus is identified in the carpal region, thumb, ring finger and the root of the little finger, with early venous return, particularly in the thumb. 
This may be done in the case of superficial malformations which are easily accessed through direct puncture under angiographic endovascular control. The transarterial, transvenous and direct puncture techniques have also been frequently used in combination to manage AVMs., ${ }^{7,17,40,41}$

Most commonly, liquid (ethanol) and semiliquid $\left(\right.$ Ony $^{\circledR}{ }^{\circledR}$, Histoacry $\left.{ }^{\circledR}\right)$ embolic agents are used. ${ }^{39,41}$

In short, a vascular malformation is a rare but severe condition that frequently requires a multidisciplinary approach.

The best results for these anomalies are achieved through the joint multidisciplinary management, control and follow-up of patients. In everyday practice, diagnostic and treatment decisions are commonly discussed among radiologists, general and plastic surgeons, dermatologists, pediatricians, trauma surgeons, hematologists, and ophthalmologists, just to name a few.

Imaging methods are the cornerstone of an adequate diagnosis and the basis of an appropriate management of these conditions. Pediatricians should be aware of these concepts to be able to guide children with such complex conditions and their families.

\section{REFERENCES}

1. Ballah D, Cahill AM, Fontalvo L, Yan A, et al. Vascular anomalies: what they are, how to diagnose them, and how to treat them. Curr Probl Diagn Radiol 2011;40(6):233-47.

2. Cahill AM, Nijs EL. Pediatric vascular malformations: pathophysiology, diagnosis, and the role of interventional radiology. Cardiovasc Intervent Radiol 2011;34(4):691-704.

3. Garzon MC, Huang JT, Enjolras O, Frieden IJ. Vascular malformations: Part I. J Am Acad Dermatol 2007;56(3):353-70.

4. Dubois J, Garel L. Imaging and therapeutic approach of hemangiomas and vascular malformations in the pediatric age group. Pediatr Radiol 1999;29(12):879-93.

5. HandJL, FriedenIJ.Vascularbirthmarksofinfancy:resolving nosologic confusion. Am J Med Genet 2002;108(4):257-64.

6. Hassanein AH, Mulliken JB, Fishman SJ, Greene AK. Evaluation of terminology for vascular anomalies in current literature. Plast Reconstr Surg 2011;127(1):347-51.

7. Legiehn GM, Heran MK. Classification, diagnosis, and interventional radiologic management of vascular malformations. Orthop Clin North Am 2006;37(3):435-74.

8. Mulliken JB, Glowacki J. Hemangiomas and vascular malformations in infants and children: a classification based on endothelial characteristics. Plast Reconstr Surg 1982;69(3):412-22.

10. JacksonIT,CarreñoR,PotparicZ,Hussain K.Hemangiomas, vascular malformations and lymphovenous malformations: classification and methods of treatment. Plast Reconstr Surg 1993;91(7):1216-30.

11. Dubois J, Alison M. Vascular anomalies: what a radiologist needs to know. Pediatr Radiol 2010;40(6):895-905.

12. Wassef M, Blei F, Adams D, Alomari A, et al. Vascular anomalies classification: recommendations from the International Society for the Study of Vascular Anomalies.
Pediatrics 2015;136(1):e203-14.

13. Enjolras O. Classification and management of the various superficial vascular anomalies: hemangiomas and vascular malformations. J Dermatol 1997;24(11):701-10.

14. Legiehn GM, Heran MK. Venous malformations: classification, development, diagnosis, and interventional radiologic management. Radiol Clin North Am 2008;46(3): 545-97.

15. Alomari A, Dubois J. Interventionalmanagement of vascular malformations. Tech Vasc Interv Radiol 2011;14(1):22-31.

16. Donnelly LF, Adams DM, Bisset GS 3rd. Vascular malformations and hemangiomas: a practical approach in a multidisciplinary clinic. AJR Am J Roentgenol 2000;174(3):597-608.

17. Moukaddam H, Pollak J, Haims AH. MRI characteristics and classification of peripheral vascular malformations and tumors. Skeletal Radiol 2009;38(6):535-47.

18. Legiehn GM, Heran MK. A step-by-step practical approach to imaging diagnosis and interventional radiologic therapy in vascular malformations. Semin Intervent Radiol 2010;27(2):209-31.

19. Garzon MC, Huang JT, Enjolras O, Frieden IJ. Vascular malformations. Part II: associated syndromes. J Am Acad Dermatol 2007;56(4):541-64.

20. Paltiel HJ, Burrows PE, Kozakewich HP, Zurakowski D, et al.Soft-tissue vascular anomalies: utility of US for diagnosis. Radiology 2000;214(3):747-54.

21. Flors L, Leiva-Salinas C, Maged IM, Norton PT, et al. MR imaging of soft-tissue vascular malformations: diagnosis, classification, and therapy follow-up. Radiographics 2011;31(5):1321-40.

22. Berenguer B, Burrows PE, Zurakowski D, Mulliken JB. Sclerotherapy of craniofacial venous malformations: complications and results. Plast Reconstr Surg 1999;104 (1):1-11.

23. Trenor CC 3rd. Sirolimus for refractory vascular anomalies. Pediatr Blood Cancer 2011;57(6):904-5.

24. Oomen KP, Paramasivam S, Waner M, Niimi Y, et al. Endoscopic transmucosal direct puncture sclerotherapy for management of airway vascular malformations. Laryngoscope 2015. Epub 2015 May 13.

25. Sierre S, Teplisky D, Garriga M, Nieto M, et al. Endoscopyassisted percutaneous treatment of laryngeal low flow vascular malformations. J Vasc Interv Radiol 2014;25(3):S129.

26. Jacobs IN, Cahill AM. Special considerations in vascular anomalies: airway management. Clin Plast Surg 2011;38(1):121-31.

27. Hassanein AH, Mulliken JB, Fishman SJ, Quatrano NA, et al. Lymphatic malformation: risk of progression during childhood and adolescence. J Craniofac Surg 2012;23(1):14952.

28. Padwa BL, Hayward PG, Ferraro NF, Mulliken JB. Cervicofacial lymphatic malformation: clinical course, surgical intervention, and pathogenesis of skeletal hypertrophy. Plast Reconstr Surg 1995;95(6):951-60.

29. Trenor CC 3rd, Chaudry G. Complex lymphatic anomalies. Semin Pediatr Surg 2014;23(4):186-90.

30. Rankin H,Zwicker K, Trenor CC. Caution is recommended prior to sildenafil use in vascular anomalies. Pediatr Blood Cancer 2015. Epub 2015 May 15.

31. Marler JJ, Mulliken JB. Current management of hemangiomas and vascular malformations. Clin Plast Surg 2005;32(1):99-116.

32. Happle R. What is a capillary malformation? J Am Acad Dermatol 2008;59(6):1077-9.

33. Greene AK, Taber SF, Ball KL, Padwa BL, et al. SturgeWeber syndrome: soft-tissue and skeletal overgrowth. J Craniofac Surg 2009;20(5):1629-30. 
34. Enjolras O, Riche MC, Merland JJ. Facial port-wine stains and Sturge-Weber syndrome. Pediatrics 1985;76(1):48-51.

35. Martí-Bonmatí L, Menor F, Mulas F. The Sturge-Weber syndrome: correlation between the clinical status and radiological CT and MRI findings. Childs Nerv Syst 1993;9(2):107-9.

36. Martí-BonmatíL, Menor F,Poyatos C, Cortina H. Diagnosis of Sturge-Weber syndrome: comparison of the efficacy of CT and MR imaging in 14 cases. AJR Am J Roentgenol 1992;158(4):867-71.

37. Liang MG,TanOT. Laser therapy of vascular malformations. In: Mulliken JB, Burrows PE, Fishman SJ, eds. MullikenE Young's Vascular Anomalies. Hemangiomas and Malformations. 2nd ed. New York: Oxford University Press; 2013. Pages 645-60.
38. Geronemus RG, Quintana AT, Lou WW, Kauvar AN. High-fluence modified pulsed dye laser photocoagulation with dynamic cooling of port-wine stains in infancy. Arch Dermatol 2000;136(7):942-3.

39. KohoutMP,Hansen M,PribazJJ,MullikenJB. Arteriovenous malformations of the head and neck: natural history and management. Plast Reconstr Surg 1998;102(3):643-54.

40. Lee BB, Do YS, Yakes W, Kim DI, et al. Management of arteriovenous malformations: a multidisciplinary approach. J Vasc Surg 2004;39(3):590-600.

41. Do YS, Yakes WF, Shin SW, Lee BB, et al. Ethanol embolization of arteriovenous malformations: interim results. Radiology 2005;235(2):674-82.

42. Yakes WF. Endovascular management of high-flow arteriovenous malformations. Semin Intervent Radiol 2004;21(1):49-58. 